\title{
Modifications to Axially Symmetric Simulations Using New DSMC (2007) Algorithms
}

\author{
Derek S. Liechty \\ Aerothermodynamics Branch, NASA Langley Research Center, Hampton, VA 23681, USA
}

\begin{abstract}
Several modifications aimed at improving physical accuracy are proposed for solving axially symmetric problems building on the DSMC (2007) algorithms introduced by Bird. Originally developed to solve nonequilibrium, rarefied flows, the DSMC method is now regularly used to solve complex problems over a wide range of Knudsen numbers. These new algorithms include features such as nearest neighbor collisions excluding the previous collision partners, separate collision and sampling cells, automatically adaptive variable time steps, a modified no-time counter procedure for collisions, and discontinuous and event-driven physical processes. Axially symmetric solutions require radial weighting for the simulated molecules since the molecules near the axis represent fewer real molecules than those farther away from the axis due to the difference in volume of the cells. In the present methodology, these radial weighting factors are continuous, linear functions that vary with the radial position of each simulated molecule. It is shown that how one defines the number of tentative collisions greatly influences the mean collision time near the axis. The method by which the grid is treated for axially symmetric problems also plays an important role near the axis, especially for scalar pressure. A new method to treat how the molecules are traced through the grid is proposed to alleviate the decrease in scalar pressure at the axis near the surface. Also, a modification to the duplication buffer is proposed to vary the duplicated molecular velocities while retaining the molecular kinetic energy and axially symmetric nature of the problem.
\end{abstract}

\section{INTRODUCTION}

With the recent introduction of modified direct simulation Monte Carlo (DSMC) algorithms ${ }^{1}$, simulations can be made more quickly using fewer numbers of simulated particles for some cases. Also, since fewer numbers of particles are required for a given flow, the range of Knudsen numbers readily simulated can be extended further into the continuum regime. The new algorithms are another step towards improving DSMC algorithms focusing on physical accuracy while improving efficiency. Some improvements in the past have included additions such as conservative species weighting ${ }^{2}$ and the use of virtual sub-cells ${ }^{3}$. However, physical improvements to the simulation of axially symmetric flows are not prevalent in the literature. One problem encountered in the calculation of the number of tentative collisions is how to define the center of the collision cell when performing collisions. If care is not taken, the number of collisions near the axis may be too high, resulting in a higher mean collision rate and lower mean free path near the axis.

Another important issue when performing axially symmetric simulations is the inclusion of a duplication buffer. The duplication buffer is used to reduce the likelihood that when a molecule moves towards the axis and is duplicated, it does not collide with a clone of itself. The clone is therefore placed in the duplication buffer and a different molecule from the buffer is placed into the flow. A modification to the duplication buffer is proposed that uses a collision cell duplication buffer rather than a global duplication buffer. This is done to promote a timeaccurate conservation of mass within the collision cells. Another modification to the cloned molecules is presented where the radial and axial velocities are unchanged while the out-of-plane velocity is reversed, thus preserving the kinetic energy of the molecule duplicated while changing nothing about the axially symmetric nature of the problem.

It will also be shown that the method by which one treats the grid and molecular movements plays an important role in the calculation of pressure along the axis. Comparisons will be made of two traditional methods to move the molecules through their respective grid scheme, each having benefits and detractors. A new, hybrid grid /molecule translation scheme will be proposed combining the benefits of both traditional schemes. Results of the new scheme will be shown to have a much better distribution of pressure along the axis than one traditional method combined 
with a lower grid merit parameter (mean collision separation divided by mean free path $(m c s / m f p)$ ) throughout the flow field compared to the other traditional method.

The baseline computations are performed using the DS2 ${ }^{4}$ code and the DSMC Analysis Code (DAC) $)^{5,6}$. However, most of the computations presented herein are results from the author's code. When a specific code is not mentioned in the text, the author is referring to his own code.

\section{TREATMENT OF AXIALLY SYMMETRIC PROBLEMS}

A complete discussion of the most common treatment of axially symmetric problems can be found in Ref. 7 . Only the relevant points to this discussion will be given in overview. One problem associated with DSMC computations for axially symmetric flows is the very small number of molecules near the axis (small volume cells) versus the large number of molecules far away from the axis (large volume cells) if the weight of each molecule is held constant. This has led to the introduction of radial weighting factors such that a simulated molecule located far from the axis represents more real molecules than one near the axis. The most recent definition of the radial weighting factor, $W_{F}$, is:

$$
W_{F}=1+r R_{W F} / y_{\max }
$$

where $r$ is the radial position of the molecule, $y_{\max }$ is the maximum radius of the flow-field, and $R_{W F}$ defines how the weighting factor varies throughout the flow field (recommended value of $10,000^{4}$ ). This results in a more uniform number of molecules per cell (for equal area cells in the $x-y$ plane), but there is now a probability that each molecule will have to be either duplicated or deleted after each movement. Because of the duplication of molecules through the use of radial weighting, there would be identical molecules present at the exact same location if a duplication buffer didn't exist. Therefore, when a molecule moves towards the axis and is duplicated, a copy of the molecule is placed in the duplication buffer and another molecule is taken from the buffer and inserted into the flow. This has traditionally been done using a global duplication buffer for the entire flow field. Weighting factors may be based either on the radius of the cell in which the molecule resides or on the radius of the molecule itself. It has been recommended ${ }^{7}$ that cell-based factors not be used. Using the traditional axially symmetric methodologies described in Ref. 7, the main issues remaining for the new DSMC (2007) algorithms are the mean collision time (mct) and pressure $(P)$ along the axis.

Throughout this paper, comparisons between different algorithms will be made using a common test case. A 10$\mathrm{cm}$ radius sphere is used in a flow of non-reacting, molecular Nitrogen where both rotational and vibrational modes have been ignored. The free stream properties are a number density of $1.2 \mathrm{e} 21 \mathrm{~m}^{-3}$, a free stream velocity of $5100 \mathrm{~m} / \mathrm{s}$ and a free stream temperature of $190 \mathrm{~K}$ resulting in a Knudsen number of 0.01 . The surface temperature was $500 \mathrm{~K}$ with the total number of simulated molecules around 600,000 . It should be noted that none of the solutions presented are grid converged within the shock layer in the sense that the maximum value of the merit parameter, in each collision cell is not on the order of 0.1 or less throughout the entire flow field. However, for gross flow field features such as local mean collision time and scalar pressure, grid convergence should not be required for reasonable results. Figure 1 presents the surface pressure computed using DS2 showing a pronounced decrease in pressure at the stagnation point $(\theta=0$-deg). DS2 implements all of the features of the modified algorithms presented in Ref. 1. Figure 2 presents the flow field $P$, mct and $m c s / m f p$ computed using DS2. This figure exemplifies the issues discussed above. The decrease in scalar pressure along the axis can be seen in Fig. 2.a. The decrease in mean collision time can be seen in Fig. 2.b. Figure 2.c presents the $m c s / m f p$. The maximum value of $m c t / m f p$ for this simulation is around 0.8 at the surface. The average value in the free-stream is on the order of 0.1-0.2. It is the purpose of the remainder of this paper to propose and examine methods to remove these non-physical behaviors for axially symmetric flows produced by the DSMC method while maintaining a relatively low merit parameter.

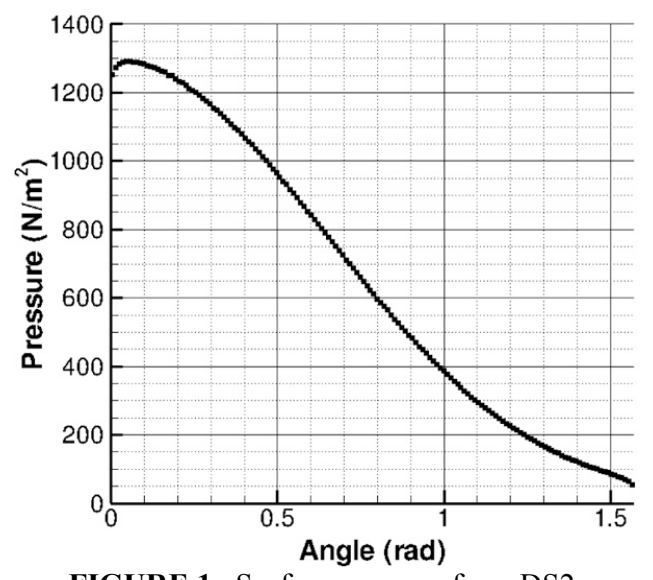

FIGURE 1. Surface pressure from DS2. 


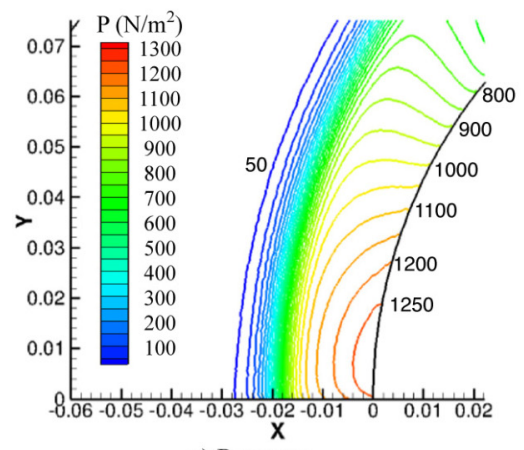

a) Pressure

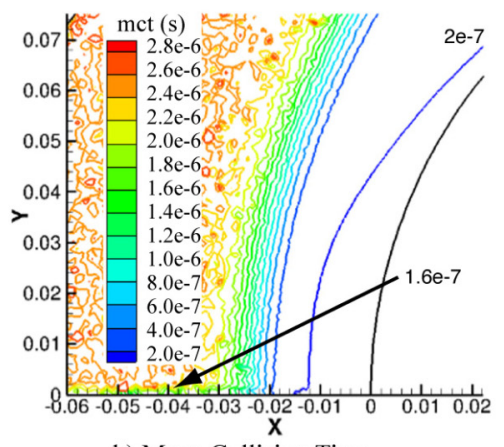

b) Mean Collision Time

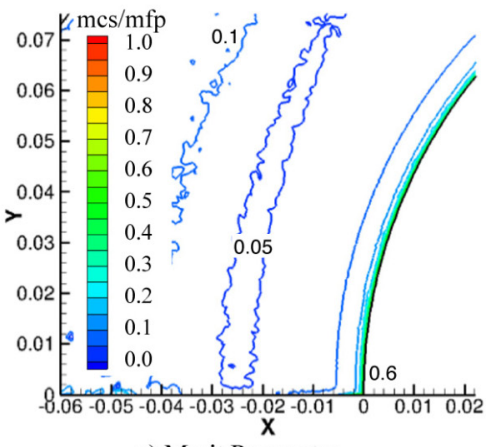

c) Merit Parameter

FIGURE 2. Solution computed by DS2.

\section{CALCULATION OF NUMBER OF TENTATIVE COLLISIONS}

The number of tentative collisions $\left(N_{C}\right)$ between similar molecules is calculated as:

$$
N_{C}=1 / 2 n(N-1)(\sigma g)_{\max } \Delta t
$$

where $n$ is the number density, $N$ is the number of molecules in the collision cell, $(\sigma g)_{\max }$ is the maximum value of the product of collision cross-section $(\sigma)$ and relative velocity $(g)$, and $\Delta t$ is the local time step. The main issue is in the definition of the number density in the collision cell. The number density can be defined as:

$$
n=N W_{F} F_{N} V_{C}^{-1}
$$

where $F_{N}$ is the global ratio of real to simulated molecules, $V_{C}$ is the volume of the collision cell, and $W_{F}$ is an average value of the radial weighting factor where all radial weights are assumed to be an average value for the collision cell. It is the definition of the center of the collision cell that can be rather ambiguous for axially symmetric simulations. There have been two traditional ways to compute the center of the collision cell:

$$
\begin{aligned}
r_{C}^{a v g} & =0.5\left(r_{\max }+r_{\min }\right) \\
r_{C}^{w t} & =\frac{2}{3} \frac{\left(r_{\text {max }}^{3}-r_{\text {min }}^{3}\right)}{\left(r_{\text {max }}^{2}-r_{\min }^{2}\right)}
\end{aligned}
$$

where $r_{\max }$ is the maximum radial coordinate of the collision cell and $r_{\min }$ is the minimum radial coordinate of the collision cell. As it turns out, both of these definitions result in the mean collision time being too small along the axis, as shown in Fig. 3. A more accurate definition of the number density at each time step can be written:

$$
n=F_{N} V_{C}^{-1} \sum_{j=1}^{N} W_{F, j}
$$
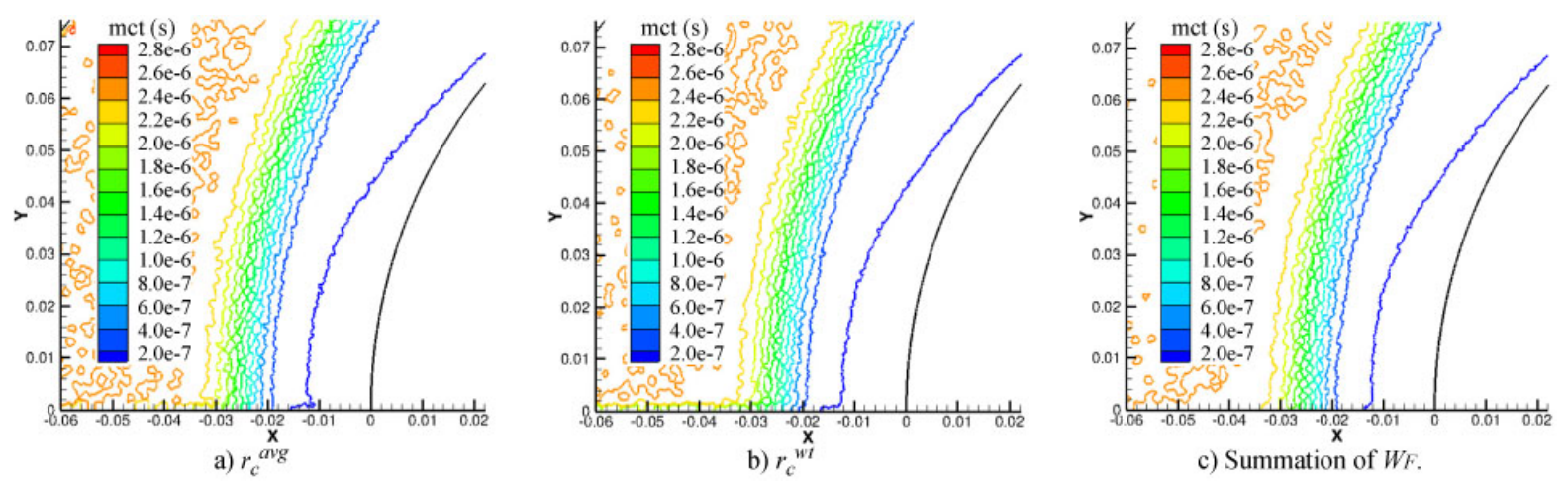

FIGURE 3. Computed mean collision times using three methodologies of computing number density within a collision cell. 
where no assumption has been made as to the weights of the molecules. The mct using eq. (6) is presented in Fig. 3.c. However, there still appears to be an error along the axis where the $m c t$ is still slightly too low. The mean collision time within a sample cell can be calculated as:

$$
m c t=\frac{1}{2} \frac{N_{\text {mols }} \Delta T_{\text {samp }}}{N_{\text {coll }} N_{\text {samp }}}
$$

where $N_{m o l s}$ is the total number of sampled molecules, $\Delta T_{\text {samp }}$ is the total sample time, $N_{\text {coll }}$ is the total number of collisions in the sample and $N_{\text {samp }}$ is the total number of samples. In equation 6 , no assumption was made as to the weights of the molecules. In equation 7, the number of molecules and number of collisions are assumed to have a constant weight within the sample cell. Therefore, it depends on how one counts the number of molecules as well as the number of collisions as to what the mean collision time is calculated to be. It is also believed that the assumption that the simulated molecules are of the same weight when collisions are performed contributes to the issues discussed herein. It is the focus of future research to include the variation of molecular weights in the collision process. For now, the mean collision time has been improved, thus the original problem has been alleviated. The result of the modified method of calculating the number of tentative collisions can be seen in Fig. 4. Although the $m c t$ has been improved, the scalar pressure along the axis remains low.

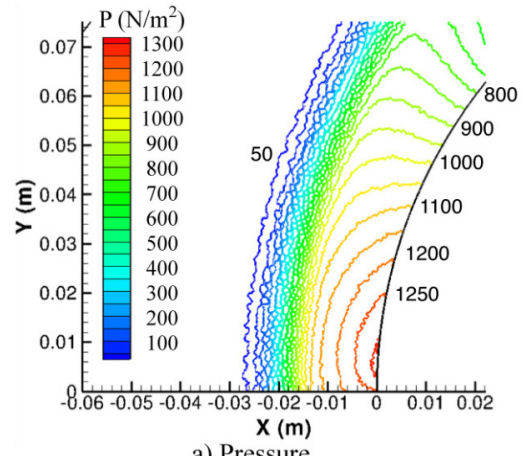

a) Pressure

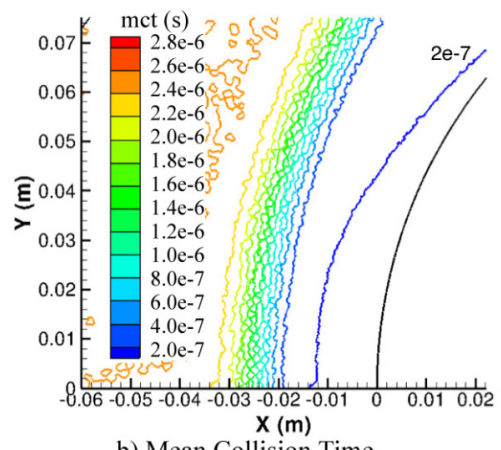

b) Mean Collision Time

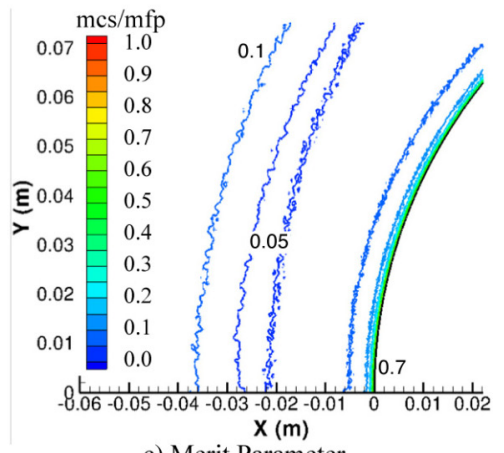

c) Merit Parameter

FIGURE 4. Solution using modified number of tentative collisions.

\section{GRID SYSTEM AND MOLECULE TRANSLATION}

There are two methods discussed presently to treat axially symmetric flows in DSMC. The first method, employed by DS2, is where the molecules are moved in a three-dimensional manner and then rotated back to the symmetry plane, accounting for the rotation in both the position and velocity ${ }^{7}$. The other method is to treat the grid as a three-dimensional wedge centered about the symmetry plane where the \pm -faces of the wedge are treated as specular reflection surfaces, thus enforcing the symmetry. However, the molecular position and velocity are never rotated back to the symmetry plane, but the $y$ - and z-velocities are changed when a reflection occurs. DAC treats axially symmetric problems in this manner with a 5-degree wedge about the symmetry plane and it is based on Bird's 1994 algorithms ${ }^{7}$, is not time accurate for grid adapted problems, and the radial weights of molecules are cellbased, not radial position-based. For the modeled conditions, pressure is rather well behaved near the axis for a grid-converged solution as shown in Fig. 5.

This grid system was has been implemented using Bird's 2007 algorithms ${ }^{1}$, now in a time-accurate implementation where the radial weighting factors vary continuously as a function of molecular position. The pressure field results of this implementation can be seen in Fig. 6.a and is markedly better along the axis than that in Fig. 4.a. However, the quality of the solution decreases as the radial position increases, especially along the surface. This is because of decreased resolution caused by the three dimensional nature of the grid. As the radial position increases, the width of the cells also increases. The values of $m c s / m f p$ are presented for this case in Fig. 6.c. Clearly, this parameter is increasing radially, resulting in a less accurate solution.

It may be that part of the improvement in pressure distribution is because

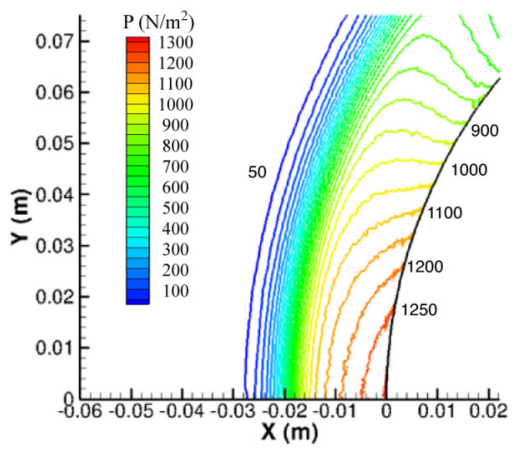

FIGURE 5. DAC pressure distribution. 


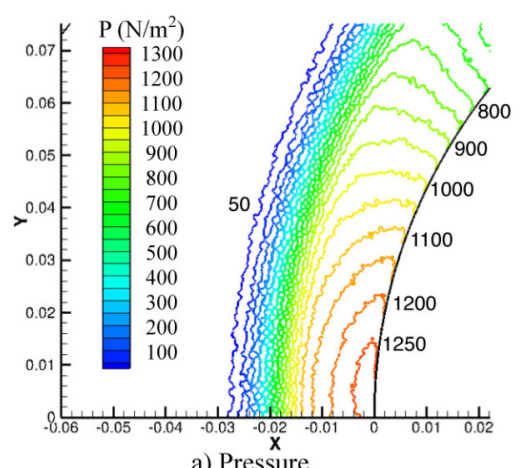

a) Pressure

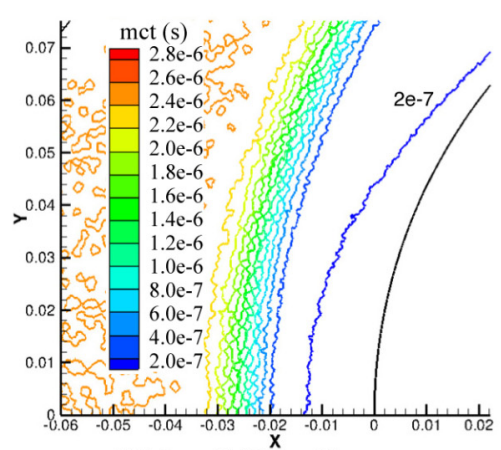

b) Mean Collision Time

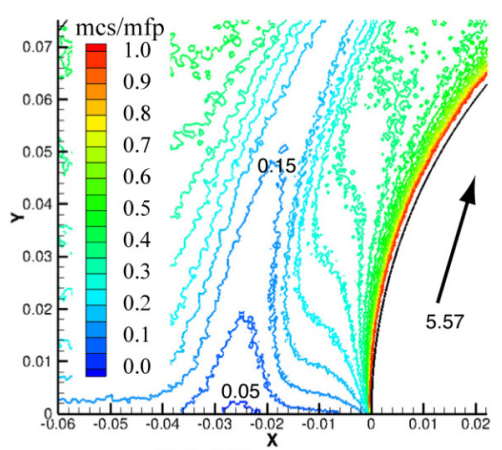

c) Merit Parameter

FIGURE 6. Results of simulation using a 5-degree wedge about the symmetry plane.

the specular reflections of the molecules reverse the component of the velocity vector perpendicular to the \pm 2.5 degree planes. Near the axis, where there may be multiple duplicates of molecules because of the radial weighting factor, this plays an important role in diversifying the population of molecules. Therefore, two changes are proposed. First, a modification to the molecule duplication process is proposed. When a molecule is duplicated, the velocity in the plane perpendicular to the radial direction and $\mathrm{x}$-axis is multiplied by $-1.0^{n c}$, where $n c$ is the cloned molecule number for multiply duplicated molecules. While this does nothing to the axially symmetric nature of the problem, it adds diversity in the population. The pressure is defined as the product of the number density, Boltzmann's constant and the translational temperature. Translational temperature is defined as:

$$
T_{t r}=\frac{1 / 2 m\left(\overline{u^{2}}+\overline{v^{2}}+\overline{w^{2}}-\bar{u}^{2}-\bar{v}^{2}-\bar{w}^{2}\right)}{3 / 2 k}
$$

Reversing the direction of the out-of-plane velocity will tend to decrease the value of $\bar{w}^{2}$, which will in turn increase the translational temperature and thus the pressure.

Secondly, another treatment of axially symmetric grid system/molecule translation is proposed to take advantage of the improvements gained by the specularly reflected treatment of molecular movement, such as with the DAC implementation. In order to decrease and thus improve the merit parameter, the angle of the reflection planes is transformed as a function of collision cell radial location. The reflection plane angle is defined as:

$$
\beta=\arcsin \left(\frac{r_{\text {max }}-r_{\text {min }}}{2 r_{\text {max }}}\right)
$$

where $r_{\max }$ and $r_{\min }$ are the maximum and minimum radial coordinates of the collision cell, such that the maximum width of the collision cell in the z-direction is equivalent to the collision cell height at the maximum angle. This gives the grid a more three dimensional appearance while limiting the width of the cells. At the end of each molecular move, since the position in the angular-direction about the axis does not affect the axially symmetric solution, the position and velocity of the molecules are rotated to a random angular location within the collision cell it resides in (the maximum and minimum defined as $\pm \beta$ ). This ensures that the molecule is within the collision cell width and there is diversity in the molecule population without affecting the physical nature of the solution. The surface pressure computed by this method is presented in Fig. 7 with a comparison to the DS2 results presented earlier. Using the modifications presented herein, there is still a slight decrease in pressure at the stagnation point, but not as pronounced. As stated earlier, it is believed that if the variation in molecular weights was accounted for in the collision process, this decrease would disappear. The flow field results are presented in Fig. 8. The pressure distribution along the axis closely resembles that of Fig. 5, and the $m c s / m f p$, while being larger than that of Fig. 4 (maximum of 1.49 versus 0.77 ), is much less than that of Fig. 6 (maximum of 1.49 versus 4.88).

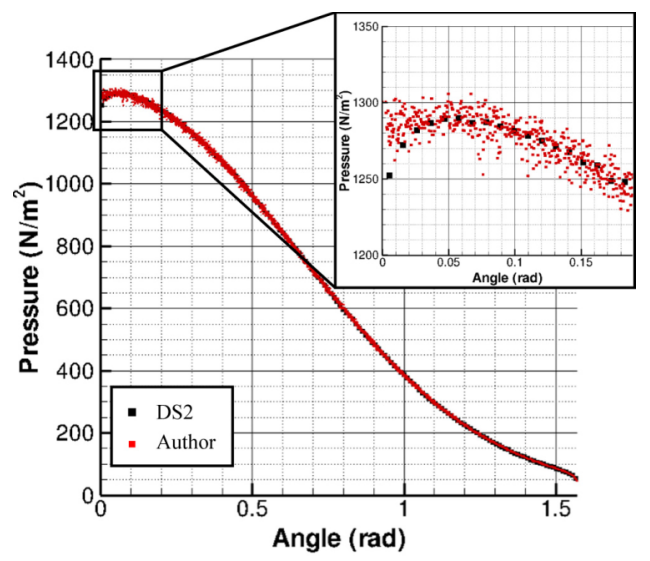

FIGURE 7. Surface pressure comparison. 


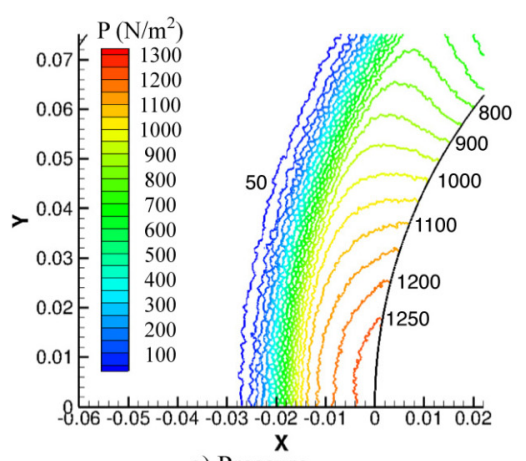

a) Pressure

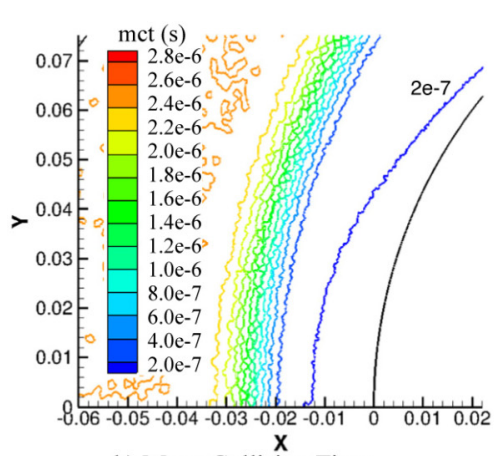

b) Mean Collision Time

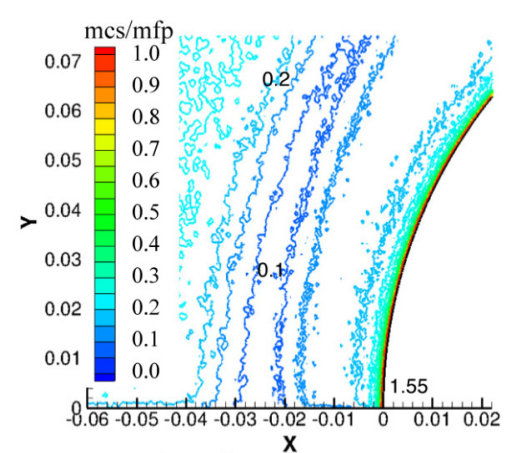

c) Merit Parameter

FIGURE 8. Solution using modified number of tentative collisions and reversal of out-of-plane velocity in duplicated molecules with specular reflection off of $\pm \beta$ planes off symmetry plane.

\section{CONCLUSION}

With the recent introduction of modified direct simulation Monte Carlo (DSMC) algorithms ${ }^{1}$, simulations can be made more quickly using fewer numbers of simulated particles for some cases. Also, since fewer numbers of particles are required for a given flow, the range of Knudsen numbers readily simulated can be extended further into the continuum regime. One problem encountered in the calculation of the number of tentative collisions is how to define the collision cell number density. For higher density simulations, the number of collisions near the axis may be too high, resulting in a higher mean collision rate and lower mean free path near the axis. A modified method to calculate the number of tentative collisions, which takes into account the varying molecular radial weights within a collision cell, has been proposed and shown to be independent of how the center of a collision cell is defined.

Another important issue when performing axially symmetric simulations is the inclusion of a duplication buffer. A modification to the cloned molecules has been presented where the radial and axial velocities are unchanged while the out-of-plane velocity is reversed, thus preserving the kinetic energy of the molecule duplicated while changing nothing about the axially symmetric nature of the problem.

It has also been shown that the method by which one treats the grid and molecular movements plays an important role in the calculation of pressure along the axis. Comparisons have been made of two traditional methods to move the molecules through their respective grid scheme, each having benefits and detractors. A new, hybrid grid /molecule translation scheme has been proposed combining the benefits of both traditional schemes. This new scheme uses reflection planes to enforce axial symmetry, as DAC does, but the reflection plane angle is a function of the collision cell radial position and height. Due to the variable reflection plane angle, the grid merit parameter has been reduced compared to the constant angle that DAC uses. Although the merit parameter is higher than that of the DS2 implementation, results of the new scheme have shown a much better distribution of pressure along the axis. While the modifications presented herein have alleviated the issues discussed above, further development of these algorithms must continue to account for the variation of molecular weights within cells.

\section{REFERENCES}

1. G. A. Bird, "Sophisticated DSMC Short Course," presented at Direct Simulation Monte Carlo Theory, Methods \& Applications conference, hosted by Sandia National Laboratory, Sept. 2007.

2. I. D. Boyd, "Conservative Species Weighting Scheme for the Direct Simulation Monte Carlo Method," Journal of Thermophysics and Heat Transfer, 10, No. 4, 579-585 (1996).

3. G. J. LeBeau, K. A. Boyles and F. E. Lumpkin III, "Virtual Sub-Cells for the Direct Simulation Monte Carlo Method," AIAA Paper 2003-1031, January 2003.

4. G. A. Bird, "Direct Simulation Monte Carlo Method," Web site: http://www.gab.com.au

5. R. G. Wilmoth, G. J. LeBeau and A. B. Carlson, "DSMC Grid Methodologies for Computing Low-Density, Hypersonic Flows About Reusable Launch Vehicles," AIAA Paper 96-1812, June 1996.

6. G. J. LeBeau and F. E. Lumkin III, "Application Highlights of the DSMC Analysis Code (DAC) Software for Simulating Rarefied Flows," Computer Methods in Applied Mechanics and Engineering, 191, No. 6-7, 595-609 (2001).

7. G. A. Bird, Molecular Gas Dynamics and the Direct Simulation of Gas Flows, Oxford University Press, Oxford, 1994. 